- Robust data collection and reporting on Fast Track discharges.

Implementation The audit data for this time period revealed a reduction in length of stay (LOS) from baseline data of six days (from 22 days to 16 days) and a reduction in admission to clinical decision to Fast Track by two days (from 15 days to 13 days) and the Fast Track process time has reduced from 7 days to 2.5 days.

Cost savings This equates to a saving of $£ 615,600$ based on the average cost of an acute bed price of $£ 300$ per day and 2052 beds saved (11 beds).

Conclusion Improvements have been made in the consistency and quality of data collection which is informing performance and evaluation of the process. Initial audit has shown that changes to how we approach Fast Track discharges have shown good progress in reduction in average length of stay and clinical decision making.

\section{P-208 A COLLABORATIVE PROJECT TO PROVIDE A RAPID PERSONALISED CARE SERVICE (RPCS) FOR PATIENTS}

${ }^{1}$ Judith Moore, ${ }^{2}$ Jackie Tritton, ${ }^{3}$ Fay Richardson. ${ }^{1}$ Rennie Grove Hospice Care, St Albans, UK; ${ }^{2}$ Peace Hospice Care, Watford, UK; ${ }^{3}$ Hospice of St Francis, Berkhamsted, UK

\subsection{6/bmjspcare-2019-HUKNC.230}

Background February 2018, the CCG reported an overspend of the CHC (Continuing Health Care) budget, with delays in funding approval and sourcing care packages, resulting in patients dying without a care package or with inappropriate hospital admissions.

Following the success of the West Hertfordshire Palliative Care Referral Centre (PCRC), (Hospice UK 2017 innovation in care award - a single point of access; three hospices and NHS community trust) three hospices collaboratively submitted a business case, proposing that any cost savings of 5\% and above would be reimbursed to the hospices. July 2018 the CCG commissioned (18-month pilot) delivering NHS CHC fast track pathway. The RPCS launched Sept 2018.

Aims of the RPCS Provide personalised care by trained end of life care carers.

- Based with the PCRC, so supporting the wider triage and coordination of Palliative/end of life care across West Herts;

- Receive hospital and community fast track referrals for community care packages

- Service provision from 8-8, 24/7 plus dedicated budget for overnight care, (sub-contracted with Marie Curie);

- More patients achieving their preferred place of care/death;

- No acute hospital discharge delays whilst waiting fast track care;

- Reduction of inappropriate hospital admission;

- Improved patient experience in last weeks of life;

- Opportunity to reach more people by the hospices.

Results to date (nine months)

- Deaths at home as a \% of all deaths has risen;

- Preferred place of death risen from $79 \%$ to $90 \%$;

- 99\% of care delivered within 48 hours;

- Only $2 \%$ of patients have exceeded the CHC fast track criteria of 12 weeks;

- $100 \%$ of care has been sourced by the hospices;

- Rise in the initiation of care packages and referrals from the previous year's data;
- Ability to change care provision at short notice ensuring patient-centred and cost effective;

- $95 \%$ of referrals met $\mathrm{CHC}$ criteria, demonstrating effective budget control.

Conclusions Based on the current figures and activity the pilot is delivering care for an increased number of patients for less care hours than previously seen in $\mathrm{CHC}$.

\section{P-209 DEVELOPMENT OF A COMMISSIONED FAST TRACK CONTINUING HEALTH CARE (CHC) SERVICE DELIVERED BY A HOSPICE}

Josephine Dedes, Ursula Reeve. St Luke's Hospice (Harrow and Brent), Harrow, UK

\subsection{6/bmjspcare-2019-HUKNC.231}

Background A review has identified the strength of the existing Hospice at Home service but weakness of contractual arrangements. The service has evolved from providing bridging to one which provides the initiation of all fast track care packages. The hospice has thus invested time and finance to support delivery of statutory CHC services.

Aims Pilot a dedicated hospice-led fast track brokerage service retaining current responsiveness and quality but delivered with $100 \%$ cost recovery for the hospice.

The pilot will respond to the NHS Long Term Plan (NHS England, 2019) and local CHC Strategic Improvement Plan (2019) requirements especially around expenditure by maximising the benefits of co-locating end of life and palliative care services.

Method Twelve month pilot will explore the:

- timeliness, quality and equity of access of a hospice-led service (Marie Curie, 2017; Marie Curie, 2019);

- interface between this service and a traditional hospice at home service;

- benefits of co-location with other hospice services;

- impact upon locality expenditure.

The service will receive, triage and assess all referrals for fast track $\mathrm{CHC}$ and initiate care.

Out of hours referrals will be picked up by the Hospice in the Home Service which will bridge care for a maximum of 72 hours.

Results A set of key performance indicators including the following will be used to measure outcomes:

- Range of packages delivered;

- \% of referrals who have care started within 24 hours of being able to receive care;

- \% of referrals that require Hospice in the Home Service to bridge care;

- $\%$ of patients utilising Personal Health Budgets.

Our intention is to demonstrate that timeliness and quality is best delivered by a hospice-led service and that this can positively impact equity of access and expenditure.

Conclusion The pilot demonstrates collaboration, has potential to positively impact patient outcomes, responds to policy requirements and could inform future commissioning recommendations. Since commencement of the pilot, initial results have identified potentially some significant benefits to patients, providers and commissioners. Therefore the poster presentation will focus on perceived outcomes. 